\title{
Glycosaminoglycans and their sulfate derivates differentially regulate the osteocytic phenotype of murine and rat osteocyte-like cell lines
}

\author{
Elena Tsourdi, Martina Rauner, Tilman D Rachner, Juliane Salbach-Hirsch, Claudia Richter and Lorenz C Hofbauer \\ Division of Endocrinology, Diabetes, and Bone Diseases, Department of Medicine III
}

Technical University, Dresden, Germany

\section{Introduction}

Extracellular matrix of bone consists primarily of collagen and glycosaminoglycans (GAGs) such as hyaluronan (HA) and chondroitin sulfate (CS). In view of the growing demand for bone replacement, collagen-GAG composites are under development for a wide range of applications in tissue engineering. Our aim was to characterize the molecular and cellular effects of GAGs on osteocytes, which are fundamental orchestrators of bone remodeling.

\section{Methods}

We manufactured native and sulfate-modified HA and CS as well as a lowmolecular weight hyaluronan LMW and evaluated their effects on viability, necrosis, apoptosis, and gene expression of osteocyte markers in the murine osteocyte-like MLO-Y4 cell line and the rat osteogenic UMR 106-01 cell line, which both display properties of primary osteocytes. Cell necrosis and apoptosis were determined using an immunoassay to detect DNA fragmentation, and cell viabilty was evaluated with a fluometric assay. The gene expression profile was examined with real-time PCR.
Native and sulfated GAGs were stable and non-cytotoxic. Assessement of native and sulfate-modified GAGs at a concentration of $200 \mu \mathrm{g} / \mathrm{ml}$ in MLO-Y4 cells demonstrated that unsulfated $\mathrm{HA}$ did not significantly reduce apoptosis compared to control, whereas the highly sulfated form of HA led to a significant reduction of apoptosis both in comparison to control and unsulfated HA $(p<0.05)$. Moreover, highly sulfated CS decreased apoptosis by $30 \%$ compared to its native form $(p<0.05)$. The LMW product did not lead to a reduction of apoptosis $(\mathbf{A})$. Similar results were observed for cell necrosis (B).

Using soluble GAGs at a concentration of $200 \mu \mathrm{g} / \mathrm{ml}$ in UMR106 cells, we found that the unsulfated form of HA dose dependently increased levels of RANKL as well as the RANKL/OPG ratio in comparison to control, whereas highly sulfated $\mathrm{HA}$ led to a significant downregulation of both levels of RANKL and the RANKL/OPG ratio when compared to its native form (both $p<0.05)(\mathbf{C}+\mathbf{D})$. These results confirm the inhibitory effect of highly sulfated HA on osteoclastogenesis which has been displayed on our previous experiments on osteoclasts.

The expression of SOST, the gene encoding sclerostin was significantly reduced by the highly sulfated HA und CS products when compared to control $(p<0.05) \quad(E)$. The expression of PHEX, a gene regulating phosphate metabolism was significantly reduced by highly sulfated HA $(p<0.05)$. Native $H A$ and both CS compounds did not alter PHEX expression (F).

\section{Conclusion}

Highly sulfated GAGs and especially HA may contribute to the phenotype of healthy, viable and functional osteocytes while maintaining a physiological RANKL/OPG ratio. The clinical significance of these findings needs to be validated in vivo.
A. Apoptosis

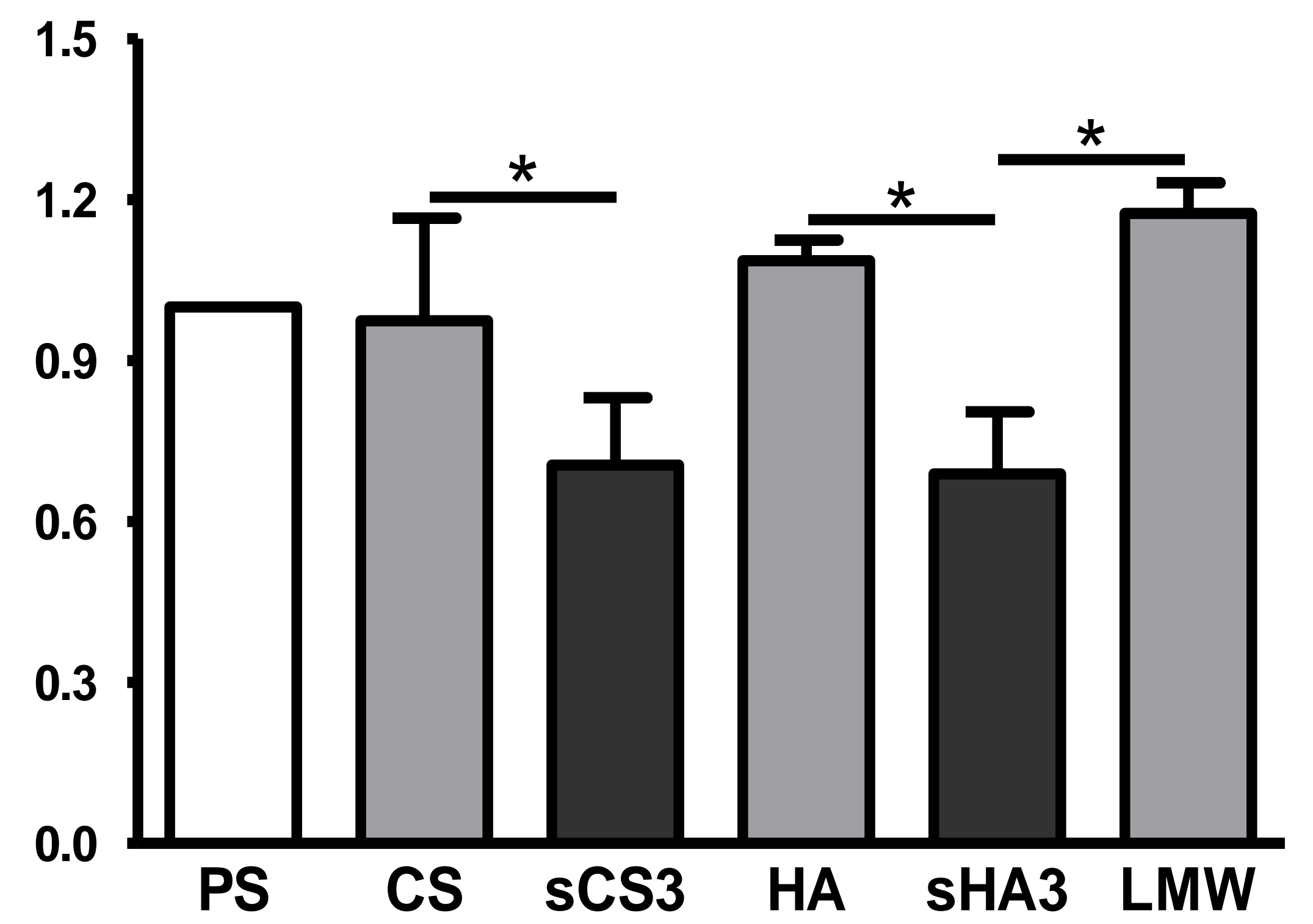

C. RANKL

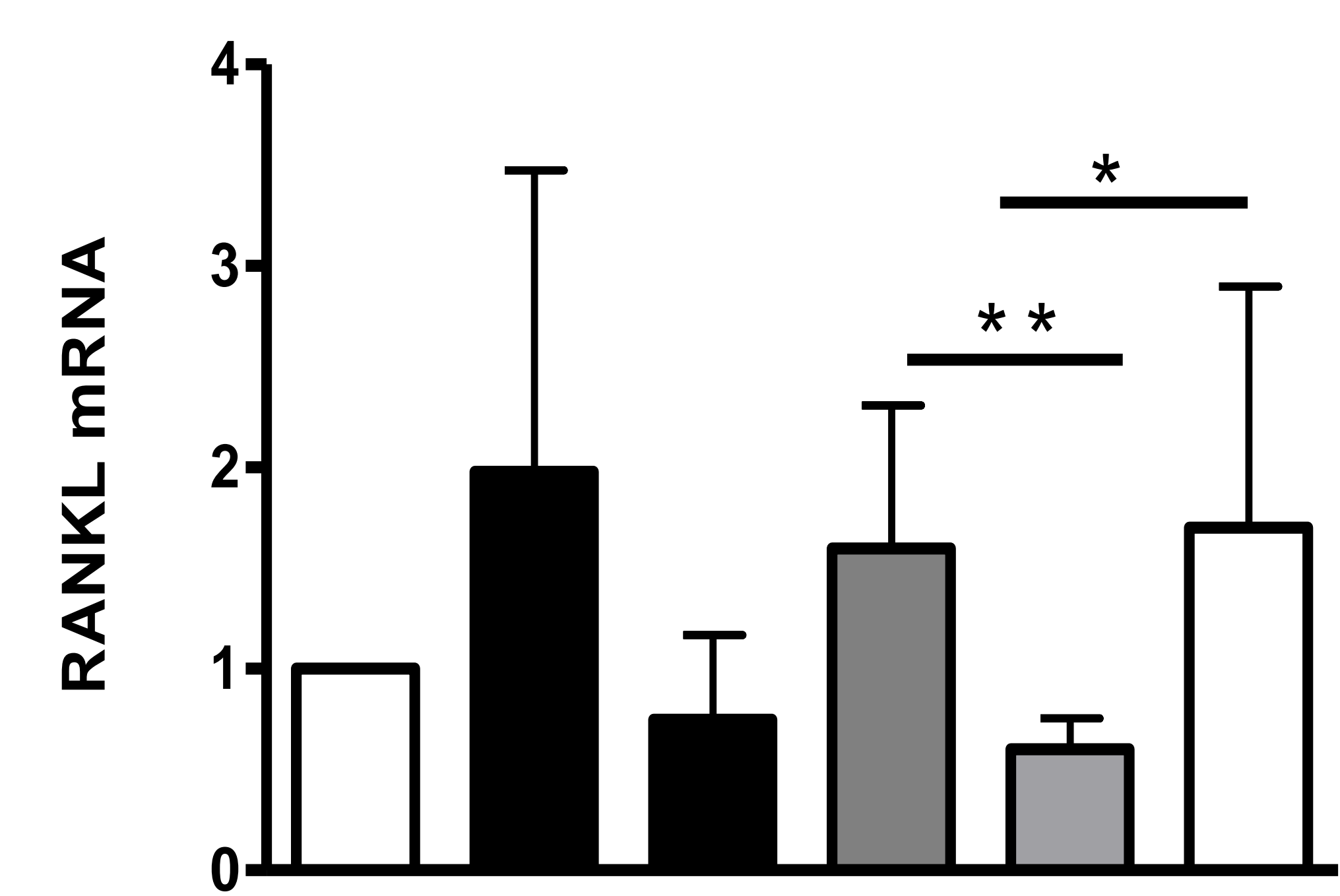

PS SCSA SCSA3 sHya SHya3 LMW

E. SOST

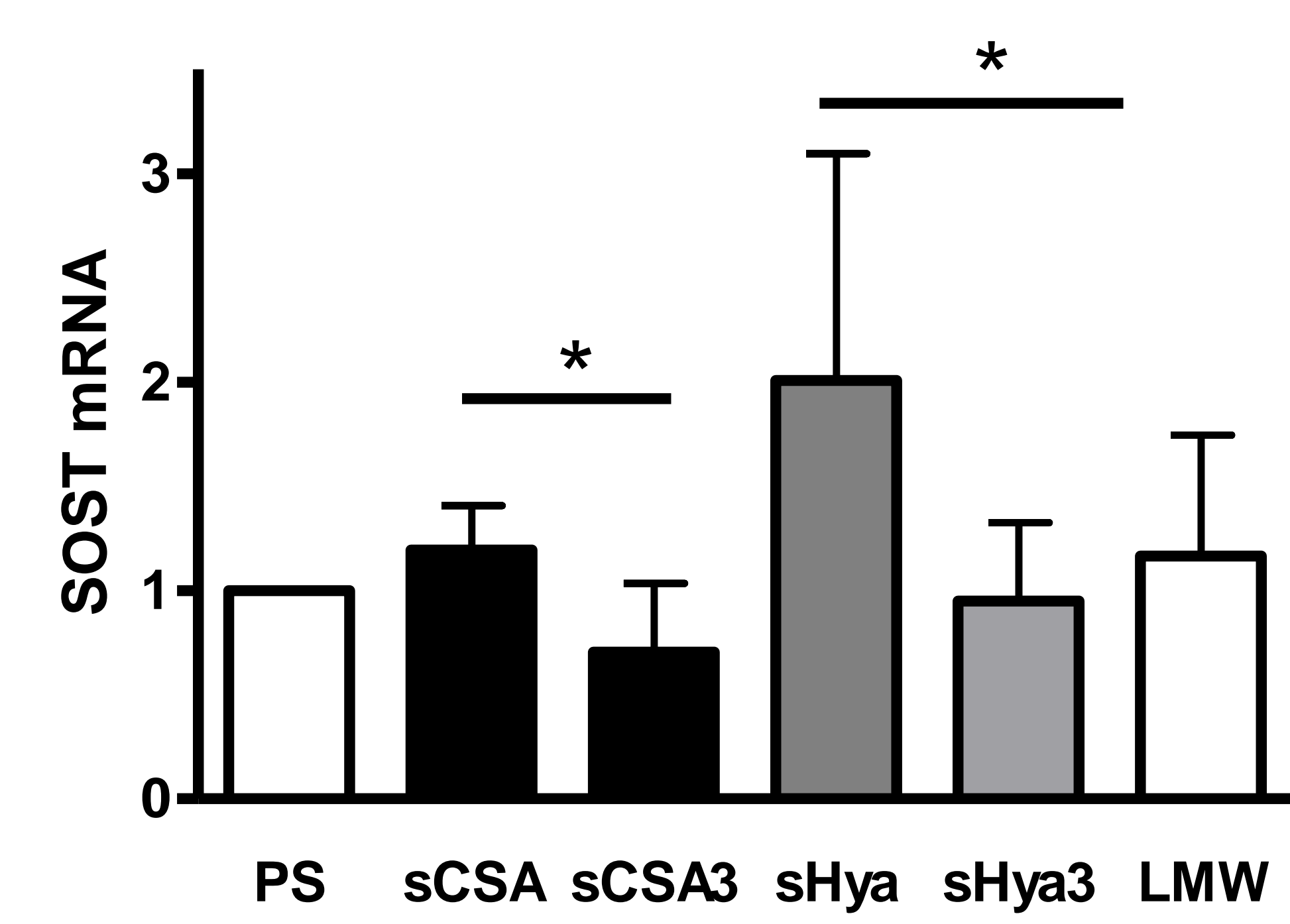

B. Necrosis

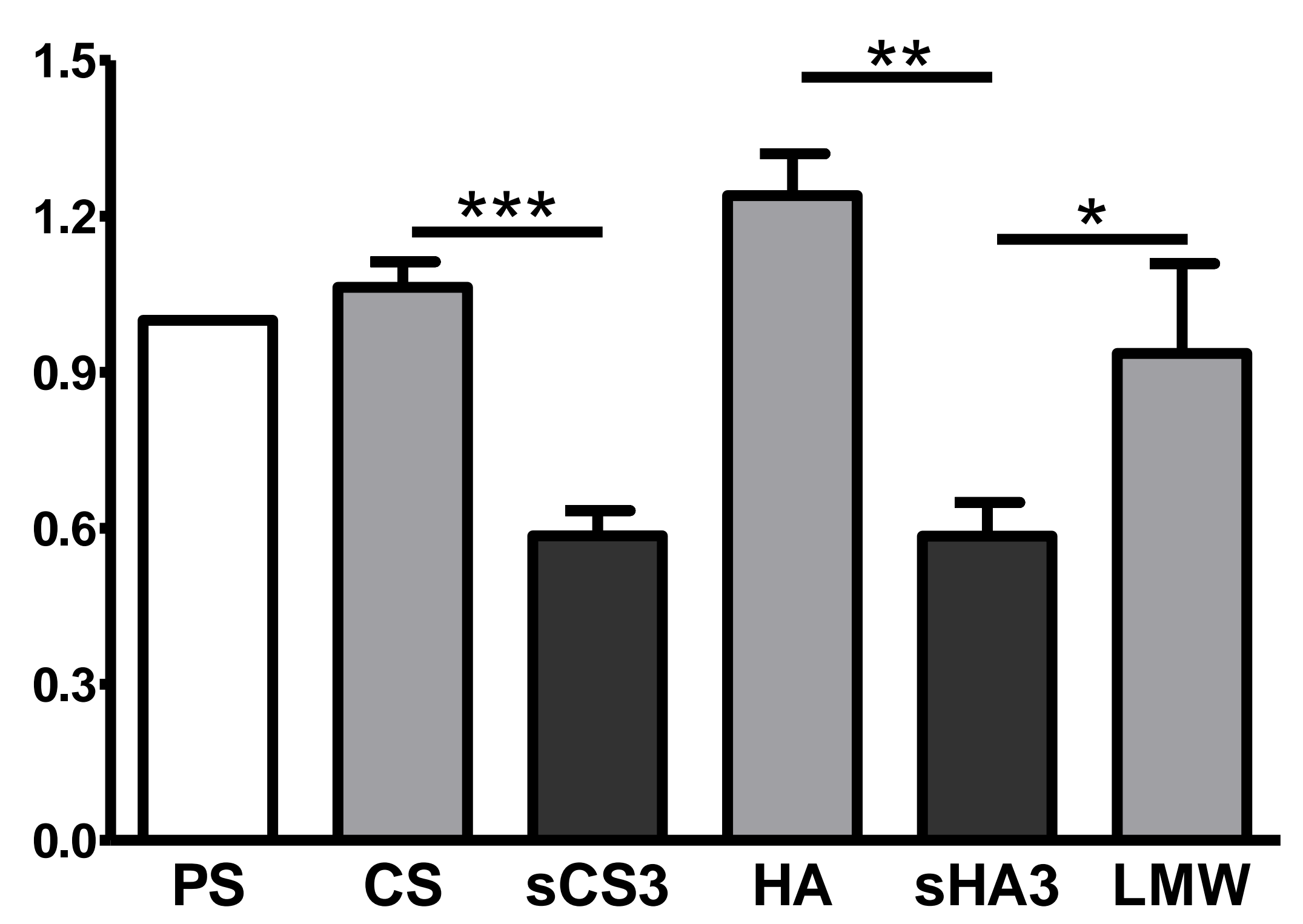

D. RANKL/OPG

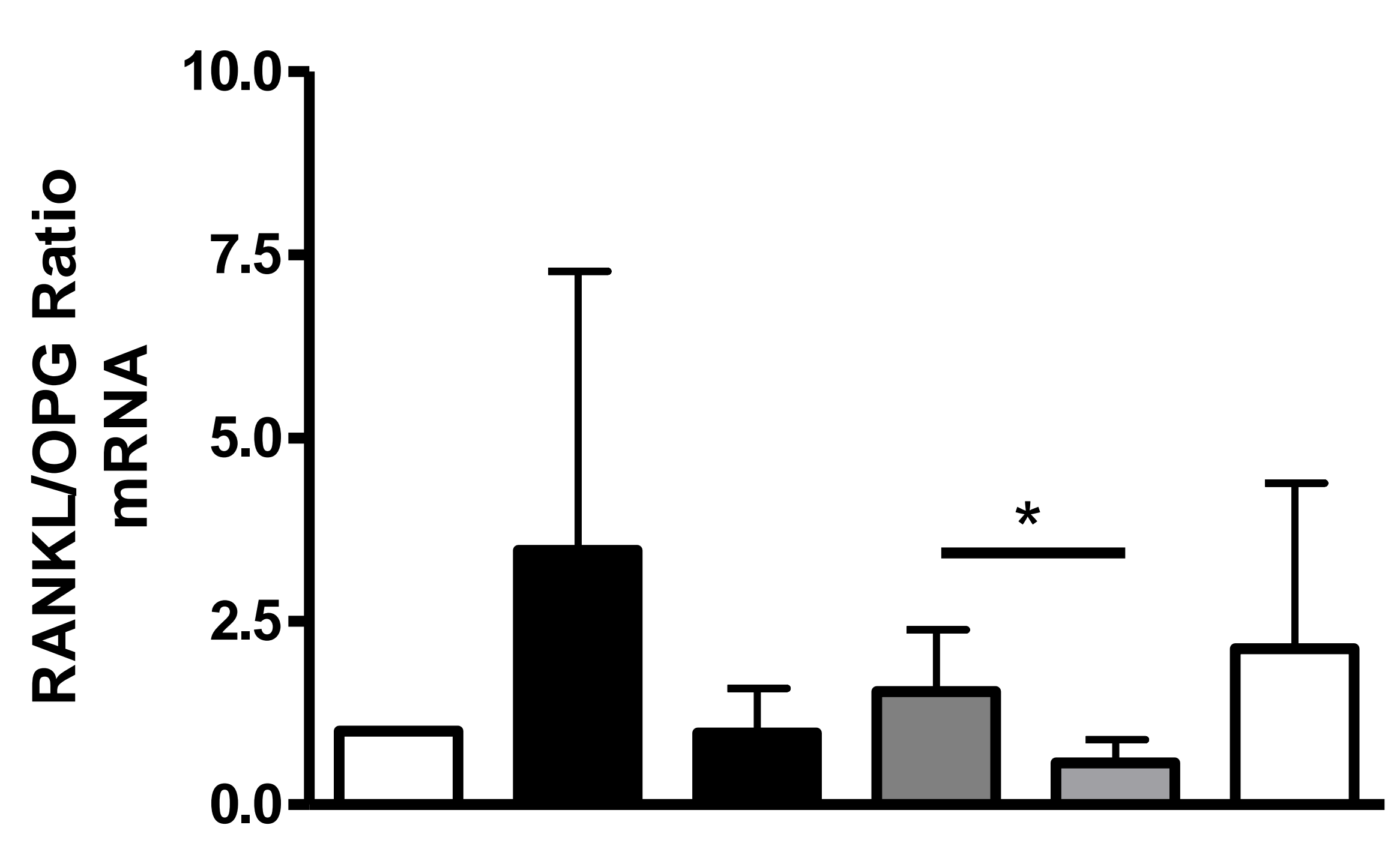

F. PHEX

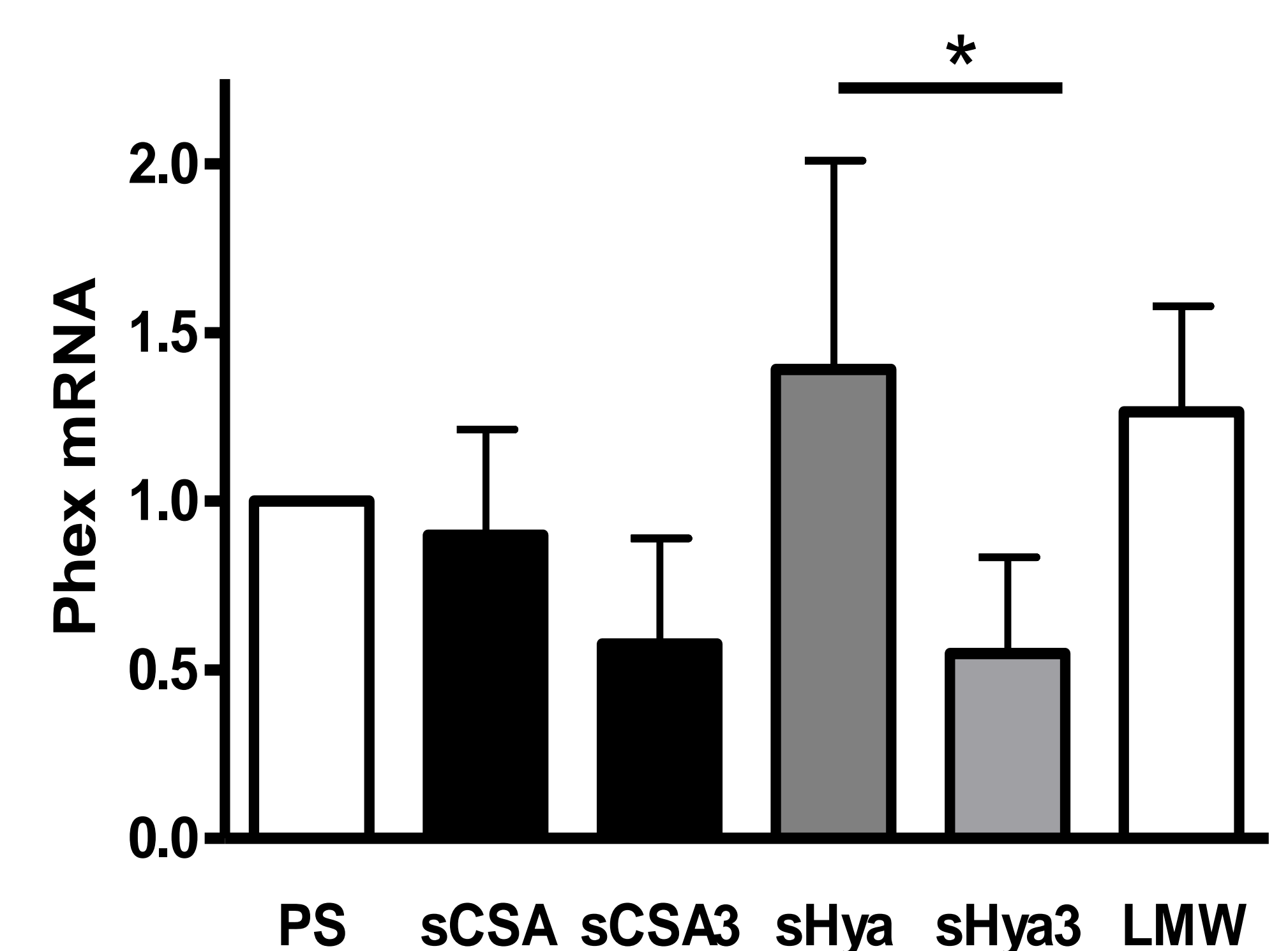

